\title{
Construction Procedure for Non-trivial T-designs
}

\author{
John Chibayi ${ }^{1}$, David Alila ${ }^{2}$, Fredrick Onyango ${ }^{1}$ \\ ${ }^{1}$ Department of Statistics and Actuarial Science, MasenoUniverisity, Nairobi, Kenya \\ ${ }^{2}$ Department of Mathematics, MasindeMuliro University of Science and Technology, Nairobi, Kenya
}

\section{Email address:}

john.chibayi@yahoo.com (J. Chibayi), aliladavid2010@gmail.com (D. Alila), onyangofredrick@hotmail.com (F. Onyango)

\section{To cite this article:}

John Chibayi, David Alila, Fredrick Onyango. Construction Procedure for Non-trivial T-designs. American Journal of Theoretical and Applied Statistics. Vol. 6, No. 1, 2016, pp. 52-60. doi: 10.11648/j.ajtas.20170601.17

Received: July 20, 2016; Accepted: August 8, 2016; Published: February 22, 2017

\begin{abstract}
A $t$-design is a generation of balanced incomplete block design (BIBD) where $\lambda$ is not restricted to the blocks in which a pair of treatments occurs but to the number of blocks in which any $t$ treatments $(t=2,3 \ldots)$ occurs. The problem of finding all parameters $\left(t, v, k, \lambda_{t}\right)$ for which $t-\left(v, k, \lambda_{t}\right)$ design exists is a long standing unsolved problem especially with $\lambda=1$ (Steiner System) as no Steiner $t$-designs are known for $t \geq 6$ when $v>k$. The objective of this study therefore to develop new methods of constructing t-designs with $t \geq 3$ and $\lambda \geq 1$. In this study $\mathrm{t}$-design is constructed by relating known BIB designs, combinatorial designs and algebraic structures with t-designs.
\end{abstract}

Keywords: Block Designs, Steiner Systems, T-designs

\section{Introduction}

$t-\left(v, k, \lambda_{t}\right)$ design is an incidence structure of points and blocks with the following properties; there are $v$ points, each block is incident with $k$ points, any point is incident with $\lambda_{1}$ blocks, and any $t$ points are incident to $\lambda_{t}$ common blocks. Where $v, k$ and $\lambda_{t}$ are all positive integers and $v \geq k \geq t$. The four numbers $t, v, k$ and $\lambda_{t}$ determine $b$ (blocks) and $\lambda_{1}$ and four numbers themselves cannot be chosen arbitrarily. For a $t-\left(v, k, \lambda_{t}\right)$ design and $S$ is any $S$-element subset of $\mathcal{S}$, with $0 \leq s \leq t$, then the number of blocks containing $S$ is given by:

$$
\lambda_{s}=\lambda \frac{\left(\begin{array}{c}
v-s \\
t-s
\end{array}\right)}{\left(\begin{array}{l}
k-s \\
t-s
\end{array}\right)} 0 \leq s \leq t
$$

particular, $=\lambda_{0}, r=\lambda_{1}, \lambda=\lambda_{t}$. Since $\lambda_{s}$ in equation $(*)$ above needs to be an integer, only the values of $v, k$ and $\lambda$ that make $\lambda_{s}$ an integer for all $0 \leq s \leq t$ are admissible parameters for a $t$-design. A tuple $\left(t, v, k, \lambda_{t}\right)$ is said to be admissible if the arithmetic conditions aforementioned hold and is said to be feasible if a $t-\left(v, k, \lambda_{t}\right)$ design exists, hence a feasible tuple is necessarily admissible [2]. The converse is not true. That is, admissibility conditions are necessary but they are not sufficient, there exist several cases of parameters that satisfy the admissibility conditions and yet no design with these parameters exists. However, it is conjectured that admissibility conditions would be sufficient, if the point set is large. This is known as $v-$ large existence conjecture or "asymptotic existence" conjecture.

The incidence structure associated with a $t$-design can be represented by a matrix. The point-block incidence matrix $A$, associated with a $t-\left(v, k, \lambda_{t}\right)$ design with $b$ blocks is a $(0-1)$ matrix of $v$ rows and $b$ columns. The elements of $A$ are $a_{i j}$ where $i$ is the point, $j$ is the block and

$$
a_{i j}=\left\{\begin{array}{c}
1 \text { if } i \in j \\
0 \text { otherwise }
\end{array}\right.
$$

There is a generalization of Fisher's inequality to $t$-designs which is due to [1]. If a $t-\left(v, k, \lambda_{t}\right)$ design exists, where $t=2 s$ is even, then the number of blocks $b \geq\left(\begin{array}{l}v \\ s\end{array}\right)$. A $t-\left(v, k, \lambda_{t}\right)$ design in which $\lambda=1$ is called Steiner system. For example a $2-(v, 3,1)$ is a Steiner triple system (STS) and a $3-(v, 4,1)$ design is a Steiner quadruple system (SQS). A $2-(v, k, \lambda)$ design is called a balanced incomplete block design (BIBD). A t-design is said to have repeated blocks if there are two blocks incident with the same set of $\mathrm{k}$ points. A $\mathrm{t}$-design with no repeated blocks is said to be simple [6].

A $t-\left(v, k, \lambda_{t}\right)$ design with $t \geq 3$ are known for only a few values of $v, k$ and $\lambda_{t}$. For $t=3$ there are several infinite families known. For instance, for any prime power $q$ and for any $d \geq 2$, there exists a $3-\left(q^{d}+1, q+1,1\right)$ design known as inversive geometry. When $d=2$, these designs 
are known as inversive planes. A Steiner quadruple system $3-(v, 4,1)$ is also known to exist for all $v \equiv 2$ or $4 \bmod 6$. Some simple $t$-designs, have been constructed for $t \leq 5$. Construction of a $6-(v, k, 1)$ design remains one of the outstanding open problems in the study of t-designs. Even for $t=4$ and $t=5$, only a few examples of $t-(v, k, 1)$ designs are known. In this study we construct some $t$-designs, with much emphasis on $\mathrm{t} \geq 3, \lambda_{t} \geq 1$ by identifying BIB designs which are also $t$-designs.

\section{Literature Review}

The main problem in $t-$ designs is the question of existence and the construction of those solutions, given admissible parameters. That is, finding all parameters $\left(t, v, k, \lambda_{t}\right)$ for which $t-\left(v, k, \lambda_{t}\right)$ design exists. There are many known Steiner $2-$ designs but constructing Steiner $t>2$ it has proved to be much harder. Wilson (1972a,1972b) building upon the work of many including ([3], [7], [10], and [11]) proved for $t=2$, fixed $k$ and sufficiently large $v$ satisfying arithmetic conditions $2-$ designs exist. There is no similar existence result yet for $t>2$. In the case of $t=3$, [4] has shown thatthere is $3-(v, 4,1)$ design if andonly if the necessary arithmetic conditions are satisfied. But for larger $k$, even $k=5$, the result is far from complete. For $\geq 3, k \geq 5$ the problem is wide open. All these constructions bear a distinct algebraic flavor in the sense that the underlying set upon which the design is constructed has a nice algebraic structure. Algebraic construction requires that a certain fixed (big) group to act as a group of automorphisms for the desired design. This technique was first formulated on paper by [11].

However this method culminates in a computer or computer-like brute-force search which cannot take us very far in quest for new $t$ - designs (especially Steiner $t$-designs). Cameron et al. [5] simplified this method, by coming up with a technique that employs transitive actions of groups. They showed that, if a group acts transitively on subsets of size the orbits for that group yield designs.

Stinson [15] came up with block spreading method for $t=2$ and for prime power index. Let $v$ be a positive integer $v \geq 2$ and let $q$ be a prime power. Suppose that there exists a $S_{q}(2, k, v)$ design satisfying $q \geq v+1$. Then there exists a group divisible design (GDD) of group type $\left(q^{d}\right)^{v}$ with block size $k$ and index one, whenever $d \geq\left(\begin{array}{l}v \\ 2\end{array}\right)$. This method has application in the construction of Steiner $2-$ designs. Blanchard ([3], [7], [14]) generalizes Hartman's [14] results for $t \geq 2$ as follows: (The "block spreading" method for $t \geq 2$ and for prime power index). Let $v$ and $t$ be positive integers, $2 \leq t \leq v$, and let $q$ be a prime power. Then there exists a number $q_{0}=q_{0}(t, v)$ such that for any $S_{q}(t, k, v)$ design satisfying $q \geq q_{0}$, there is a $t$-GDD of group type $\left(q^{d}\right)^{v}$ with block size $k$ and index one whenever $d \geq\left(\begin{array}{l}\mathrm{v} \\ \mathrm{t}\end{array}\right)$. More so, ([5], [8]) generalizes Blanchard's construction for general index (the "block spreading" method for $t \geq 2$ and general index). Let $v, t$ and $\lambda$ be a positive integers $2 \leq t \leq$ $v$. Then there exists a number $q_{0}=q_{0}(t, v)$ such that for any $S_{\lambda}(t, k, v)$ design with prime power decomposition $\lambda=$ $q_{1}, q_{2}, q_{3} \cdots q_{n}$ satisfying $q_{i} \geq q_{0} ; 1 \leq i \leq n$; there is a $t$-GDD of group type $\left(\lambda^{d}\right)^{v}$ with block size $k$ and index one whenever $d \geq\left(\begin{array}{l}\mathrm{v} \\ \mathrm{t}\end{array}\right)$. This generalized "block spreading" construction has several application such as constructing new Steiner 3 -designs and new group divisible $t$-designs with index one. Limitation of this method is that the bounds on $d$ are too large.

Magliveras et al. [9] constructed some new large sets of tdesigns, using recursive construction described by Qiu-rong $\mathrm{Wu}$ [14]. Wu [14] showed that if there exists large sets $L S(n)-\left(t, k, v_{1}\right), L S(n)-\left(t, k, v_{2}\right), L S(n)-(k-2, k-$ $\left.1, v_{1}-1\right), L S(n)-\left(k-2, k-1, v_{2}-1\right)$, then there exists a large set $L S(n)-\left(t, k, v_{1}+v_{2}-k+1\right)$. He went on to show that also, if there exist a large sets $L S(n)-(t, v, k)$ and $L S(n)-(k-2, k-1, v-1)$ then there exist large sets $L S(n)-(t, k, v+m(v-k+1))$ for all $m \geq 0$.

Mohácsy and Ray-Chaudhuri [12] constructed $t$-designs from known $t$-wise balanced designs. In his works he showed that, given a positive integer $k$ and a $t-$ $\left(v,\left(k_{1}, k_{2} \cdots k_{s}\right), \lambda\right)$ design $D$, with all blocks-sizes $k_{i}$ occurring in $D$ and $1 \leq t \leq k \leq k_{1} \leq k_{2} \cdots \leq k_{s}$, the construction produces a $t-(v, k, n \lambda)$ design $D^{*}$, with $n=L . C . M\left[\left(\begin{array}{c}k_{1}-t \\ k-t\end{array}\right), \cdots\left(\begin{array}{c}k_{s}-t \\ k-t\end{array}\right)\right]$. Onyango [13] on his part constructed $t$-designs with $t=3$ and $\lambda=1$ from balanced incomplete block design.

\section{Construction of Some $t-\left(v, k, \lambda_{t}\right)$ Designs with $t=3$ and $\lambda_{t}=1$}

The properties of $t-\left(v, k, \lambda_{t}\right)$ designs include:

$$
\begin{gathered}
b k=\lambda_{1} v \\
\lambda_{t}(v-(t-1))=\lambda_{t-1}(k-(t-1))
\end{gathered}
$$

Replacing $t=3$ and $\lambda_{t}=1$ in equation (2) we have:

$$
v-2=\lambda_{2}(k-2) \Rightarrow \lambda_{2}=\frac{v-2}{k-2}
$$

Now when $t=2$ we have:

$$
\lambda_{2}(v-1)=\lambda_{1}(k-1) \Rightarrow \frac{\lambda_{1}}{\lambda_{2}}=\frac{v-1}{k-1}
$$

This implies

$$
\lambda_{1}=\lambda_{2} \frac{(v-1)}{(k-1)} ; \lambda_{1}=\propto(v-1) \lambda_{2}=\lambda_{1} \frac{(k-1)}{(v-1)} ; \lambda_{2}=\propto(k-1)
$$

Given that $\lambda_{1}, \lambda_{2}, v-1$ andk -1 are all integers and $\propto$ is a rational number which we will represent by $\frac{x}{y}$ where $x a n d y$ are positive integers. Thus the equations (5) become:

$$
y \lambda_{1}=x(v-1) \text { and } y \lambda_{2}=x(k-1)
$$

Case 1: When $x=1$

Then (6) becomes: $y \lambda_{1}=v-1, \Longrightarrow v=y \lambda_{1}+1$

$$
y \lambda_{2}=k-1, \Rightarrow k=y \lambda_{2}+1
$$

Theorem 1: If $x=1$ and $\lambda_{2}-1 \equiv 0(\bmod y)$, where $y$ is 
an integer then there are only three non- trivial $3-(v, k, 1)$ designs which are: $3-(8,4,1), 3-(22,6,1)$ and $3-$ $(112,12,1)$

Proof: From (2), (3), and (7)

$$
\lambda_{2}=\frac{y \lambda_{1}+1-2}{y \lambda_{2}+1-2}=\frac{y \lambda_{1}-1}{y \lambda_{2}-1}
$$

This implies: $\lambda_{1}=\lambda^{2}{ }_{2}-\frac{\lambda_{2}-1}{y} v=y \lambda^{2}{ }_{2}-\lambda_{2}+2$ and $k=y \lambda_{2}+1$. For this design to be $3-(v, k, 1)$ design and from (1) it implies

$$
\left(\lambda^{2}{ }_{2}-\frac{\lambda_{2}-1}{y}\right)\left(y \lambda^{2}{ }_{2}-\lambda_{2}+2\right) \equiv 0 \bmod \left(y \lambda_{2}+1\right)
$$

That is:

$$
\frac{\left(y \lambda^{2}{ }_{2}-\lambda_{2}+1\right)\left(y \lambda^{2}{ }_{2}-\lambda_{2}+2\right)}{y\left(y \lambda_{2}+1\right)}
$$

which is a positive integer. Expanding and simplifying equation (9), we obtain

$$
\lambda^{3}{ }_{2}-\frac{3 \lambda^{2} 2}{y}+\frac{\lambda_{2}(3 y+4)}{y^{2}}-\frac{6 y+4}{y^{3}}+\frac{2 y^{2}+6 y+4}{y^{2}\left(y^{2} \lambda_{2}+y\right)}
$$

The last term of equation (10), that is

$$
\frac{2 y^{2}+6 y+4}{y^{2}\left(y^{2} \lambda_{2}+y\right)}
$$

will be an integer if $y^{2}$ divides $6 y+4$. The only values for $y$ in which this is possible are 1 and 2. In this case Equation (11) is not an integer. Thus both Equations (10) and (11) will be integers if $\lambda_{2}$ takes the values $2,3,5$ and 11 . The table below gives corresponding values of $k, \lambda_{1}$, vand $b$.

Table 1. Case1; for $y=1$ the possible cases of $3-(v, k, 1)$ designs.

\begin{tabular}{lllll}
\hline $\boldsymbol{\lambda}_{\mathbf{2}}$ & $\boldsymbol{k}$ & $\boldsymbol{\lambda}_{\mathbf{1}}$ & $\boldsymbol{v}$ & $\boldsymbol{b}$ \\
\hline 2 & 3 & 3 & 4 & 4 \\
3 & 4 & 7 & 8 & 14 \\
5 & 6 & 21 & 22 & 77 \\
11 & 12 & 111 & 112 & 1036 \\
\hline
\end{tabular}

The required $3-(v, k, 1)$ designs are; $3-(8,4,1), 3-$ $(22,6,1)$, and $3-(112,12,1)$. A $3-(4,3,1)$ is trivial given $t=k$, but it is required that $t<k$, hence is not included and our proof is completed.

Case 2: When $y=1$

In this case Equation (6) becomes

$$
\lambda_{1}=x(v-1), \Rightarrow v=\frac{\lambda_{1}+x}{x} \text { and } \lambda_{2}=x(k-1), \Rightarrow k=\frac{\lambda_{2}+x}{x}
$$

Where is $x$ a positive integer and both $\lambda_{1}$ and $\lambda_{2}$ are divisible by $x$.

From Equation (1) we get $\lambda_{1}, v$, and $k$ as follows;

$$
\lambda_{1}=\lambda^{2}{ }_{2}-x \lambda_{2}+x, v=\frac{\lambda^{2}{ }_{2}-x \lambda_{2}+2 x}{x}
$$

and

$$
k=\frac{\lambda_{2}+x}{x}
$$

Using $b=\frac{\lambda_{1} v}{k}$ for this design to be $3-(v, k, 1)$ design then:

$$
\left(\lambda^{2}{ }_{2}-x \lambda_{2}+x\right) \frac{\left(\lambda^{2}{ }_{2}-x \lambda_{2}+2 x\right)}{x} \equiv 0 \bmod \left(\frac{\lambda_{2}+x}{x}\right)
$$

That is

$$
\frac{\left(\lambda^{2}{ }_{2}-x \lambda_{2}+x\right)\left(\lambda^{2}{ }_{2}-x \lambda_{2}+2 x\right)}{\lambda_{2}+x}
$$

which is a positive integer. Expanding and simplifying equation (14), we obtain

$$
\lambda^{3}{ }_{2}-3 x \lambda^{2}{ }_{2}+\lambda_{2}\left(3 x+4 x^{2}\right)-\left(6 x^{2}+4 x^{3}\right)+\frac{2 x^{2}+6 x^{3}+4 x^{4}}{\lambda_{2}+x}
$$

Equation (15) will be and integer ifthe last term $\frac{2 x^{2}+6 x^{3}+4 x^{4}}{\lambda_{2}+x}$ is an integer. Thus $\lambda_{2}$ can take any of the following values $3,4,6,8,10,18,28,32,38,58$, and 118 . But $\lambda_{2}$ must be divisible by 2 . So 3 is not a possibility. We give corresponding values of $k, \lambda_{1}, v$ and $b$ in the Table 2 below.

Table 2. Case 2; for $x=2$ the possible cases of $3-(v, k, 1)$ designs.

\begin{tabular}{lllll}
\hline $\boldsymbol{\lambda}_{\mathbf{2}}$ & $\boldsymbol{k}$ & $\boldsymbol{\lambda}_{\mathbf{1}}$ & $\boldsymbol{v}$ & $\boldsymbol{b}$ \\
\hline 4 & 3 & 10 & 6 & 20 \\
6 & 4 & 26 & 14 & 91 \\
8 & 5 & 50 & 26 & 260 \\
10 & 6 & 82 & 42 & 574 \\
18 & 10 & 290 & 146 & 4234 \\
22 & 12 & 442 & 222 & 8177 \\
28 & 15 & 730 & 366 & 17812 \\
38 & 20 & 1370 & 686 & 46991 \\
58 & 30 & 3250 & 1626 & 176150 \\
118 & 60 & 13690 & 6846 & 1562029 \\
\hline
\end{tabular}

Thefollowing designs $3-(6,3,1), 3-(14,4,1), 3-$ $(26,5,1), 3-(42,6,1), 3-(146,10,1), 3-(222,12,1), 3-$ $(366,15,1), 3-(686,20),, 3-(1676,30,1)$ and $3-$ $(6846,60,1)$. can then be obtain from $\operatorname{BIB}(v, k, \lambda)$ designs given below

$$
\begin{aligned}
2-(6,3,4), 2- & (14,4,6), 2-(26,5,8), 2-(42,6,10), 2 \\
& -(146,10,18), 2-(222,12,22), 2 \\
& -(366,15,28), 2-(686,20,38), 2 \\
& -(1626,30,58), \text { and } 2-(6846,60,118) .
\end{aligned}
$$

For $x=3$, the possible values are: $6,9,15,18,21,33,39,60,69,81,123,165,249$, and 501. The corresponding values of $\lambda_{1}, v, k$, and $b$ in the Table 3 below.

Table 3. Case2; for $x=3$ the possible cases of $3-(v, k, 1)$ designs.

\begin{tabular}{lllll}
\hline $\boldsymbol{\lambda}_{\mathbf{2}}$ & $\boldsymbol{k}$ & $\boldsymbol{\lambda}_{\mathbf{1}}$ & $\boldsymbol{v}$ & $\boldsymbol{b}$ \\
\hline 6 & 3 & 21 & 8 & 56 \\
9 & 4 & 57 & 20 & 285 \\
15 & 6 & 183 & 62 & 1891 \\
18 & 7 & 273 & 92 & 3588 \\
21 & 8 & 381 & 128 & 6096 \\
33 & 12 & 993 & 332 & 27473 \\
39 & 14 & 1407 & 470 & 47235 \\
60 & 21 & 3423 & 1142 & 186146 \\
69 & 24 & 4557 & 1520 & 288610 \\
\hline
\end{tabular}




\begin{tabular}{lllll}
\hline $\boldsymbol{\lambda}_{\mathbf{2}}$ & $\boldsymbol{k}$ & $\boldsymbol{\lambda}_{\mathbf{1}}$ & $\boldsymbol{v}$ & $\boldsymbol{b}$ \\
\hline 81 & 28 & 6321 & 2108 & 475881 \\
165 & 56 & 26733 & 8912 & 4254366 \\
249 & 84 & 61257 & 20420 & 14891285 \\
501 & 168 & 249501 & 83168 & 123514876 \\
\hline
\end{tabular}

Again we obtain $3-(v, k, 1)$ designs from BIB designs below:

$$
\begin{aligned}
2-(8,3,6), 2- & (20,4,9), 2-(67,6,15), 2-(92,7,18), 2 \\
& -(128,8,21), 2-(332,12,33), \ldots, \text { and } 2 \\
& -(83168,168,501)
\end{aligned}
$$

For $x=4$, and using similar arguments as before we obtain possible values of $\lambda_{2}$ as follows: $8,12,16,20,28,32,36, \ldots$. which give the values of $\lambda_{1}, k, v$ and $b$ as follows:

Table 4. Case2; for $x=4$ the possible cases of $3-(v, k, 1)$ designs.

\begin{tabular}{lllll}
\hline $\boldsymbol{\lambda}_{\mathbf{2}}$ & $\boldsymbol{k}$ & $\boldsymbol{\lambda}_{\mathbf{1}}$ & $\mathbf{V}$ & $\mathbf{B}$ \\
\hline 8 & 3 & 36 & 10 & 120 \\
12 & 4 & 100 & 26 & 650 \\
16 & 5 & 196 & 50 & 1960 \\
20 & 6 & 324 & 82 & 4428 \\
28 & 8 & 676 & 170 & 14365 \\
32 & 9 & 900 & 226 & 22600 \\
36 & 10 & 1156 & 290 & 33524 \\
\hline
\end{tabular}

The desired $3-(v, k, 1)$ designs are obtained from the following BIB designs:

$$
\begin{aligned}
2-(10,3,8), 2 & -(26,4,12), 2-(50,5,16), 2-(82,6,20), 2 \\
& -(170,8,28), 2-(226,9,32), \\
& \text { and } 2-(290,10,36)
\end{aligned}
$$

Remark: this construction can go on and on by simply varying the values of $x a n d y$ for each case, but as values of xandy increases the designs obtained have large parameters making them not practical.

\section{Construction of $t-\left(v, k, \lambda_{t}\right)$ Designs with $t=3$ and $\lambda_{t} \geq 1$}

We extend the work in [10] by constructing $3-$ designs with $\lambda_{t} \geq 1$, that is for general indexand Steiner 4 -designs. When $t=3, \lambda_{t}=c$ and from Equation (2) we have:

$$
\begin{aligned}
& \lambda_{1}=\frac{\lambda_{2}(v-1)}{k-1} ; \Rightarrow \lambda_{1}=\propto(v-1) \text { and } \\
& \lambda_{2}=\frac{\lambda_{1}(k-1)}{v-1} ; \Rightarrow \lambda_{2}=\propto(k-1)
\end{aligned}
$$

Where $\propto$ is a rational number since $\lambda_{1}, \lambda_{2}, c, v-$ 1 and $k-1$ are all positive integers hence, we will represent it by $\frac{x}{y}$ where $x$ and $y$ are positive integers.

Case I, $x=1$

Then Equation (15) becomes:

$$
\lambda_{2}=\frac{c\left(y \lambda_{1}-1\right)}{y \lambda_{2}-1} \lambda_{1}=\frac{y \lambda^{2}{ }_{2}-\lambda_{2}+c}{c y} \text { and }=\frac{y \lambda^{2}{ }_{2}-\lambda_{2}+2 c}{c}
$$

For this design to be $3-(v, k, c)$ design and from $b k=v \lambda_{1} ;$ it implies

$$
\frac{\left(y \lambda^{2}{ }_{2}-\lambda_{2}+c\right)}{c y}\left(\frac{y \lambda^{2}{ }_{2}-\lambda_{2}+2 c}{c}\right) \equiv 0 \bmod \left(y \lambda_{2}+1\right)
$$

That is,

$$
\frac{\left(y \lambda^{2}{ }_{2}-\lambda_{2}+c\right)\left(y \lambda^{2}{ }_{2}-\lambda_{2}+2 c\right)}{c^{2} y\left(y \lambda_{2}+1\right)}
$$

Expanding and simplification of Equation (19)we obtain

$$
\frac{\lambda^{3} 2}{c^{2}}-\frac{3 \lambda^{2}{ }_{2}}{c^{2} y}+\frac{\lambda_{2}(3 c y+4)}{c^{2} y^{2}}-\frac{(6 c y+4)}{c^{2} y^{3}}+\frac{2 c^{2} y^{2}+6 c y+4}{c^{2} y^{2}\left(y^{2} \lambda_{2}+y\right)}
$$

Which will be an integer if $c^{2} y^{2}$ divides $6 c y+4$. For $c=2$, that is $\lambda_{t}=2$, the only possible values for $y$ in which this is possible are 1 and 2. Thus Equation (20) will be an integer if $\lambda_{2}$ takes only of the following values; 2 and 5 . The table below gives corresponding values of $\lambda_{1}, k, v$ and $b$

Table 5. Case 1; for $y=1$ and $c=2$ the possible cases of $3-(v, k, c)$ designs.

\begin{tabular}{lllll}
\hline$\lambda_{2}$ & $\boldsymbol{k}$ & $\boldsymbol{\lambda}_{\mathbf{1}}$ & $\boldsymbol{v}$ & $\boldsymbol{b}$ \\
\hline 2 & 3 & 2 & 3 & 2 \\
5 & 6 & 11 & 12 & 22 \\
\hline
\end{tabular}

The first $3-(3,3,2)$ does not exist hence we have only one $3-(v, k, c)$ design in this case.

This $3-(12,6,2)$ is identified with this BIB design $B(12,6,5)$.

CASE II, $y=1$

In this case Equation (17) becomes:

$$
\lambda_{2}=\frac{c\left(\lambda_{1}-x\right)}{\lambda_{2}-x} \lambda_{1}=\frac{\lambda^{2}{ }_{2}-x \lambda_{2}+c x}{c} \text { and } v=\frac{\lambda^{2}{ }_{2}-x \lambda_{2}+2 c x}{c x}
$$

Using similar argument as before:

$$
\frac{\left(\lambda_{2}^{2}-x \lambda_{2}+x c\right)\left(\lambda_{2}^{2}-x \lambda_{2}+2 c x\right)}{c^{2}\left(\lambda_{2}+x\right)}
$$

Will be integer if Equation (22) is and integer

$$
\frac{2 c^{2} x^{2}+6 c x^{3}+4 x^{4}}{c^{2}\left(\lambda_{2}+x\right)}
$$

We give the corresponding values of $\lambda_{2}, \lambda_{1}, v, k$ and $b$ in Table 6 below;

Table 6. Case 2; for $x=2$ and $c=2$ the possible cases of $3-(v, k, c)$ designs.

\begin{tabular}{lllll}
\hline $\boldsymbol{\lambda}_{\mathbf{2}}$ & $\boldsymbol{k}$ & $\boldsymbol{\lambda}_{\mathbf{1}}$ & $\boldsymbol{v}$ & $\boldsymbol{b}$ \\
\hline 4 & 3 & 6 & 4 & 8 \\
6 & 4 & 14 & 8 & 28 \\
10 & 6 & 42 & 22 & 154 \\
14 & 8 & 86 & 44 & 473 \\
22 & 12 & 222 & 112 & 2072 \\
46 & 24 & 1014 & 508 & 21463 \\
\hline
\end{tabular}

The following designs; $3-(4,3,2), 3-(8,4,2), 3-$ $(22,6,2), 3-(44,8,2), 3-(112,12,2)$, and $3-(508,24,2)$ can be obtained from $B I B(v, k, \lambda)$ designs given below: $B(4,3,4), B(8,4,6), B(22,6,10), B(44,8,14), B(112,12,22)$, and $B(508,24,46)$ For $c=2, x=3$ and using similar 
arguments as before we give the values of $\lambda_{2}, \lambda_{1}, v, k a n d b$ as in Table 7 below:

Table 7. Case 2; for $x=3$ and $c=2$ the possible cases of $3-(v, k, c)$ designs.

\begin{tabular}{lllll}
\hline $\boldsymbol{\lambda}_{\mathbf{2}}$ & $\boldsymbol{k}$ & $\boldsymbol{\lambda}_{\mathbf{1}}$ & $\mathbf{V}$ & $\mathbf{B}$ \\
\hline 6 & 3 & 12 & 5 & 20 \\
12 & 5 & 57 & 20 & 228 \\
15 & 6 & 93 & 32 & 496 \\
27 & 10 & 327 & 110 & 3597 \\
\hline
\end{tabular}

We get the desired $3-(v, k, c)$ designs from BIB designs below:

$$
B(5,3,6), B(20,5,12), B(32,6,15) \text { and }(110,10,27)
$$

For $c=2, x=4$ and using the same methods we give the table of values of; $\lambda_{2}, \lambda_{1}, k, v$ and $b$ as follows:

Table 8. Case 2; for $x=4$ and $c=2$ the possible cases of $3-(v, k, c)$ designs.

\begin{tabular}{lllll}
\hline $\boldsymbol{\lambda}_{\mathbf{2}}$ & $\boldsymbol{k}$ & $\boldsymbol{\lambda}_{\mathbf{1}}$ & $\boldsymbol{v}$ & $\boldsymbol{b}$ \\
\hline 8 & 3 & 20 & 6 & 40 \\
12 & 4 & 52 & 14 & 182 \\
16 & 5 & 100 & 26 & 520 \\
20 & 6 & 168 & 42 & 1126 \\
36 & 10 & 580 & 146 & 8468 \\
44 & 12 & 884 & 222 & 16354 \\
56 & 15 & 1460 & 366 & 35624 \\
\hline
\end{tabular}

Similarly, for $c=2, x=5$ the values of $\lambda_{1}, \lambda_{2}, k, v$ and $b$ we give them as in the Table 9 below:

Table 9. Case 2; for $x=5$ and $c=2$ the possible cases of $3-(v, k, c)$ designs.

\begin{tabular}{|c|c|c|c|c|}
\hline$\lambda_{2}$ & $\boldsymbol{k}$ & $\lambda_{1}$ & $v$ & $b$ \\
\hline 10 & 3 & 30 & 7 & 70 \\
\hline 20 & 5 & 155 & 32 & 992 \\
\hline 25 & 6 & 255 & 52 & 2210 \\
\hline 30 & 7 & 380 & 77 & 4180 \\
\hline 65 & 14 & 1955 & 392 & 54740 \\
\hline
\end{tabular}

Now, for $c=3, x=3$ and $x=6$ and applying the same methods, we give values of $\lambda_{2}, \lambda_{1}, v, k$ and $b$ in table 10 below respectively.

Table 10. Case 2; for $x=3$ and $c=3$ the possible cases of $3-(v, k, c)$ designs.

\begin{tabular}{lllll}
\hline $\boldsymbol{\lambda}_{\mathbf{2}}$ & $\boldsymbol{k}$ & $\boldsymbol{\lambda}_{\mathbf{1}}$ & $\boldsymbol{v}$ & $\boldsymbol{b}$ \\
\hline 6 & 3 & 9 & 4 & 12 \\
9 & 4 & 21 & 8 & 42 \\
15 & 6 & 63 & 22 & 231 \\
24 & 9 & 171 & 58 & 1102 \\
51 & 18 & 819 & 274 & 12467 \\
\hline
\end{tabular}

Table 11. Case 2; for $x=6$ and $c=3$ the possible cases of $3-(v, k, c)$ designs.

\begin{tabular}{|c|c|c|c|c|}
\hline$\lambda_{2}$ & $\boldsymbol{k}$ & $\lambda_{1}$ & $v$ & $b$ \\
\hline 12 & 3 & 30 & 6 & 60 \\
\hline 18 & 4 & 78 & 14 & 273 \\
\hline 24 & 5 & 150 & 26 & 780 \\
\hline 30 & 6 & 246 & 42 & 1722 \\
\hline
\end{tabular}

Also for $c=4, x=2, x=4$ and $x=6$ and using similar arguments, we give values of $\lambda_{2}, \lambda_{1}, v, k$, and $b$ in the Tables below respectively.

Table 12. Case 2; for $x=2$ and $c=4$ the possible cases of $3-(v, k, c)$ designs.

\begin{tabular}{|c|c|c|c|c|}
\hline$\lambda_{2}$ & $\boldsymbol{k}$ & $\lambda_{1}$ & $v$ & $b$ \\
\hline 4 & 3 & 4 & 3 & 4 \\
\hline 6 & 4 & 8 & 5 & 10 \\
\hline 10 & 6 & 22 & 12 & 44 \\
\hline 22 & 12 & 112 & 57 & 532 \\
\hline
\end{tabular}

The first $3-(3,3,4)$ design is trivial.

Table 13. Case 2; for $x=4$ and $c=4$ the possible cases of $3-(v, k, c)$ designs.

\begin{tabular}{lllll}
\hline $\boldsymbol{\lambda}_{\mathbf{2}}$ & $\boldsymbol{k}$ & $\boldsymbol{\lambda}_{\mathbf{1}}$ & $\boldsymbol{v}$ & $\boldsymbol{b}$ \\
\hline 12 & 4 & 28 & 8 & 56 \\
20 & 6 & 84 & 22 & 308 \\
28 & 8 & 172 & 44 & 946 \\
\hline
\end{tabular}

Table 14. Case 2; for $x=6$ and $c=4$ the possible cases of $3-(v, k, c)$ designs.

\begin{tabular}{|c|c|c|c|c|}
\hline$\lambda_{2}$ & $\boldsymbol{k}$ & $\lambda_{1}$ & $v$ & $b$ \\
\hline 18 & 4 & 60 & 11 & 165 \\
\hline 24 & 5 & 114 & 20 & 456 \\
\hline 30 & 6 & 186 & 32 & 992 \\
\hline
\end{tabular}

When $c=5, x=5$ and $x=10$ and using similar methods the values of $\lambda_{2}, \lambda_{1}, v, k$ and $b$ we give them in the tables below respectively.

Table 15. Case 2; for $x=5$ and $c=5$ the possible cases of $3-(v, k, c)$ designs.

\begin{tabular}{lllll}
\hline $\boldsymbol{\lambda}_{\mathbf{2}}$ & $\boldsymbol{k}$ & $\boldsymbol{\lambda}_{\mathbf{1}}$ & $\boldsymbol{v}$ & $\boldsymbol{b}$ \\
\hline 15 & 4 & 35 & 8 & 70 \\
20 & 5 & 65 & 14 & 182 \\
25 & 6 & 105 & 22 & 385 \\
45 & 10 & 365 & 74 & 2701 \\
\hline
\end{tabular}

Table 16. Case 2; for $x=10$ and $c=5$ the possible cases of $3-(v, k, c)$ designs.

\begin{tabular}{lllll}
\hline $\boldsymbol{\lambda}_{\mathbf{2}}$ & $\boldsymbol{k}$ & $\boldsymbol{\lambda}_{\mathbf{1}}$ & $\boldsymbol{v}$ & $\boldsymbol{b}$ \\
\hline 30 & 4 & 130 & 14 & 455 \\
40 & 5 & 250 & 26 & 1300 \\
50 & 6 & 410 & 42 & 2870 \\
\hline
\end{tabular}

or $c=6, x=3$ and $x=6$ we get the following tables respectively.

Table 17. Case 2; for $x=3$ and $c=6$ the possible cases of $3-(v, k, c)$ designs.

\begin{tabular}{lllll}
\hline $\boldsymbol{\lambda}_{\mathbf{2}}$ & $\boldsymbol{k}$ & $\boldsymbol{\lambda}_{\mathbf{1}}$ & $\boldsymbol{v}$ & $\boldsymbol{b}$ \\
\hline 15 & 6 & 33 & 12 & 66 \\
24 & 9 & 87 & 30 & 290 \\
51 & 18 & 411 & 138 & 3151 \\
\hline
\end{tabular}

Table 18. Case 2; for $x=6$ and $c=6$ the possible cases of $3-(v, k, c)$ designs

\begin{tabular}{lllll}
\hline $\boldsymbol{\lambda}_{\mathbf{2}}$ & $\boldsymbol{k}$ & $\boldsymbol{\lambda}_{\mathbf{1}}$ & $\boldsymbol{v}$ & $\boldsymbol{b}$ \\
\hline 18 & 4 & 42 & 8 & 84 \\
30 & 6 & 126 & 22 & 462 \\
42 & 8 & 258 & 44 & 1419 \\
\hline
\end{tabular}


Also for $c=7$ and $x=7$ we get the following table:

Table 19. Case 2; for $x=7$ and $c=7$ the possible cases of $3-(v, k, c)$ designs.

\begin{tabular}{lllll}
\hline $\boldsymbol{\lambda}_{\mathbf{2}}$ & $\boldsymbol{k}$ & $\boldsymbol{\lambda}_{\mathbf{1}}$ & $\boldsymbol{v}$ & $\boldsymbol{b}$ \\
\hline 21 & 4 & 49 & 8 & 98 \\
35 & 6 & 147 & 22 & 539 \\
42 & 7 & 217 & 32 & 992 \\
\hline
\end{tabular}

Lastly, for $c=8, x=4$ and $x=8$ we also get the following tables respectively.

Table 20. Case 2; for $x=4$ and $c=8$ the possible cases of $3-(v, k, c)$ designs.

\begin{tabular}{lllll}
\hline $\boldsymbol{\lambda}_{\mathbf{2}}$ & $\boldsymbol{k}$ & $\boldsymbol{\lambda}_{\mathbf{1}}$ & $\boldsymbol{v}$ & $\boldsymbol{b}$ \\
\hline 12 & 4 & 16 & 5 & 20 \\
20 & 6 & 44 & 12 & 88 \\
28 & 8 & 88 & 23 & 253 \\
44 & 12 & 224 & 57 & 1064 \\
\hline
\end{tabular}

Table 21. Case 2; for $x=8$ and $c=8$ the possible cases of $3-(v, k, c)$ designs.

\begin{tabular}{lllll}
\hline $\boldsymbol{\lambda}_{\mathbf{2}}$ & $\boldsymbol{k}$ & $\boldsymbol{\lambda}_{\mathbf{1}}$ & $\boldsymbol{v}$ & $\boldsymbol{b}$ \\
\hline 24 & 4 & 56 & 8 & 112 \\
40 & 6 & 168 & 22 & 616 \\
56 & 8 & 344 & 44 & 1892 \\
88 & 12 & 888 & 112 & 8288 \\
\hline
\end{tabular}

Case III, $x \neq$ yand $x>1, y>1$

Letting

$$
\lambda_{1}=\frac{x}{y}(v-1) \Rightarrow v=\frac{y \lambda_{1}+x}{x} \operatorname{and} \lambda_{2}=\frac{x}{y}(k-1) \Rightarrow k=\frac{y \lambda_{2}+x}{x}
$$

Where $x$ and $y$ are positive integers and $\lambda_{2}$ and $y$ are divisible by $x$ and substituting these values of $v$ and $k$ in:

$$
\lambda_{2}=\frac{c(v-2)}{k-2}
$$

We obtain $\lambda_{1}, v$, and $k$ as follows:

$$
\lambda_{1}=\frac{y \lambda^{2}{ }_{2}-x \lambda_{2}+c x}{c y}, v=\frac{y \lambda^{2}{ }_{2}-x \lambda_{2}+2 c x}{c x} \text { and } k=\frac{y \lambda_{2}+x}{x}
$$

Using $b=\frac{\lambda_{1} v}{k}$ for this to be $3-(v, k, 1)$ design then

$$
\begin{gathered}
\frac{\left(y \lambda^{2}{ }_{2}-x \lambda_{2}+c x\right)}{c y}\left(\frac{y \lambda_{2}^{2}-x \lambda_{2}+2 c x}{c x}\right) \\
\equiv 0 \bmod \left(\frac{y \lambda_{2}+x}{x}\right)
\end{gathered}
$$

That is Equation (24) is a positive integer

$$
\frac{\left(y \lambda^{2}{ }_{2}-x \lambda_{2}+c x\right)\left(y \lambda^{2}{ }_{2}-x \lambda_{2}+2 c x\right)}{c^{2} y\left(y \lambda_{2}+x\right)}
$$

Expanding and simplifying Equation (24) we obtain

$$
\begin{gathered}
y \lambda^{3}{ }_{2}-3 x \lambda^{2}{ }_{2}+\frac{\lambda^{2}{ }_{2}\left(3 c x y+4 x^{2}\right)}{y}-\frac{\left(6 c x^{2} y+4 x^{3}\right)}{y^{2}}+ \\
\frac{2 c^{2} x^{2} y^{2}+6 c x^{3} y+4 x^{4}}{c^{2} y^{3}\left(y \lambda_{2}+x\right)}
\end{gathered}
$$

Under this case and using similar method we find $3-$ $(v, k, c)$ exists if Equation (26) is an integer:

$$
\frac{2 c^{2} x^{2} y^{2}+6 c x^{3} y+4 x^{4}}{c^{2} y^{3}\left(y \lambda_{2}+x\right)}=\frac{2 x^{2}\left(c^{2}+\frac{3 c x}{y}+\frac{2 x^{2}}{y^{2}}\right)}{c^{2} y\left(y \lambda_{2}+x\right)}
$$

Taking $c=5, x=5$ and $y=2$ in this case there is only one non-trivial $3-(v, k, 5)$ and $\lambda_{2}$ would take the values 5 or 35 with corresponding values of $k, \lambda_{1}$, vandbas in the table below.

Table 22. Case 3; for $x=5, y=2$ and $c=5$ the possible cases of $3-$ $(v, k, c)$ designs.

\begin{tabular}{lllll}
\hline $\boldsymbol{\lambda}_{\mathbf{2}}$ & $\boldsymbol{k}$ & $\boldsymbol{\lambda}_{\mathbf{1}}$ & $\boldsymbol{v}$ & $\boldsymbol{b}$ \\
\hline 5 & 3 & 5 & 3 & 5 \\
35 & 15 & 230 & 93 & 1426 \\
\hline
\end{tabular}

For $c=7, x=7$ and $y=2$, we get the following table:

Table 23. Case 3; for $x=7, y=2$ and $c=7$ the possible cases of $3-$ $(v, k, c)$ designs.

\begin{tabular}{lllll}
\hline $\boldsymbol{\lambda}_{2}$ & $\boldsymbol{k}$ & $\boldsymbol{\lambda}_{\mathbf{1}}$ & $\boldsymbol{v}$ & $\boldsymbol{b}$ \\
\hline 7 & 3 & 7 & 3 & 7 \\
21 & 7 & 56 & 17 & 136 \\
\hline
\end{tabular}

\section{Construction of $4-(v, k, 1)$ Designs}

The same technique that has been used to construct $3-(v, k, 1)$ is applied. When $t=4$ and $\lambda_{t}=1$, we have;

$$
\lambda_{2}=\propto(v-2) \text { and } \lambda_{3}=\propto(k-2)
$$

Given $\lambda_{1}, \lambda_{2}, \lambda_{3}, v-2, v-1, k-2$, and $k-1$ are all integers, and $\propto$ is a rational number which we will represent by $\frac{x}{y}$ where $x$ and $y$ are positive integers.

Case $1 x=1$

Equation (28) becomes;

$y \lambda_{2}=v-2, \Rightarrow v=y \lambda_{2}+2 \operatorname{and} y \lambda_{3}=k-2, \Rightarrow k=y \lambda_{3}+2$

Using Equation (29) and (30) we obtain

$$
\begin{gathered}
\lambda_{1}=\frac{y \lambda_{2}{ }^{2}+\lambda_{2}}{y \lambda_{3}+1} \\
\lambda_{1}=\frac{y^{2} \lambda_{3}{ }^{4}-2 y \lambda^{3}{ }_{3}+3 y \lambda^{2}{ }_{3}+\lambda^{2}{ }_{3}-3 \lambda_{3}+2}{y \lambda_{3}+1}
\end{gathered}
$$

Which implies;

$$
v=y \lambda^{2}{ }_{3}-\lambda_{3}+3 \text { and } k=y \lambda_{3}+2
$$

For this design to be $4-(v, k, 1)$ and from $b k=v \lambda_{1}$ it means

$$
\begin{gathered}
\frac{\left(y^{2} \lambda_{3}{ }^{4}-2 y \lambda^{3}{ }_{3}+3 y \lambda^{2}{ }_{3}+\lambda^{2}{ }_{3}-3 \lambda_{3}+2\right)\left(y \lambda^{2}{ }_{3}-\lambda_{3}+3\right)}{\left(y \lambda_{3}+1\right)} \\
\equiv 0 \bmod \left(y \lambda_{3}+2\right)
\end{gathered}
$$

Hence Equation (31) is a positive integer

$$
\frac{\left(y^{2} \lambda_{3}{ }^{4}-2 y \lambda^{3}{ }_{3}+3 y \lambda^{2}{ }_{3}+\lambda^{2}{ }_{3}-3 \lambda_{3}+2\right)\left(y \lambda^{2}{ }_{3}-\lambda_{3}+3\right)}{\left(y \lambda_{3}+1\right)\left(y \lambda_{3}+2\right)}
$$


Expanding Equation (31) we obtain

$$
\begin{gathered}
\frac{y^{2} \lambda_{3}{ }^{5}}{\left(y \lambda_{3}+1\right)}-\frac{5 y \lambda_{3}{ }^{4}}{\left(y \lambda_{3}+1\right)}+\frac{\lambda_{3}{ }^{3}\left(6 y^{2}+13 y\right)}{y\left(y \lambda_{3}+1\right)} \\
-\frac{\lambda_{3}{ }^{2}\left(24 y^{2}+27 y\right)}{y^{2}\left(y \lambda_{3}+1\right)} \\
+\frac{\lambda_{3}\left(11 y^{3}+54 y^{2}+54 y\right)}{y^{3}\left(y \lambda_{3}+1\right)} \\
-\frac{\left(33 y^{3}+108 y^{2}+108\right)}{y^{4}\left(y \lambda_{3}+1\right)}+\frac{6 y^{4}+66 y^{3}+216 y^{2}+216 y}{y^{4}\left(y \lambda_{3}+1\right)\left(y \lambda_{3}+2\right)}
\end{gathered}
$$

Equation (32) will be integer whenever Equation (33) is an integer

$$
\frac{6 y^{4}+66 y^{3}+216 y^{2}+216 y}{y^{4}\left(y \lambda_{3}+1\right)\left(y \lambda_{3}+2\right)}
$$

Using Equation (33), corresponding values of $\lambda_{1}, \lambda_{2}, k, v$ and $b$ will be generated as shown in Table 12:

Table 24. Case 1; for $y=1$ the possible cases of $4-(v, k, 1)$ designs.

\begin{tabular}{llllll}
\hline$\lambda_{3}$ & $\lambda_{2}$ & $\lambda_{1}$ & $\boldsymbol{k}$ & $\boldsymbol{v}$ & $\boldsymbol{b}$ \\
\hline 2 & 3 & 4 & 4 & 5 & 5 \\
5 & 21 & 77 & 7 & 23 & 253 \\
\hline
\end{tabular}

The first $4-(5,4,1)$ design is trivial. The $4-(23,7,1)$ is the only non trivial. This $4-(v, k, 1)$ is then identified with the following BIB designs; $2-(5,4,3)$ and $2-(23,7,21)$

Case 2, $y=1$

In this case Equation (29) becomes

$$
\begin{aligned}
& \lambda_{1}=\frac{\lambda_{3}{ }^{4}-2 x \lambda^{3}{ }_{3}+3 x \lambda^{2}{ }_{3}+x^{2} \lambda^{2}{ }_{3}-3 x^{2} \lambda_{3}+2 x^{2}}{\lambda_{3}+x} v= \\
& \frac{\lambda_{3}{ }^{2}-x \lambda_{3}+3 x}{x} \text { and } k=\frac{\lambda_{3}+2 x}{x}
\end{aligned}
$$

For this design to be $4-(v, k, 1)$ and from $b k=v \lambda_{1}$ it means

$$
\begin{gathered}
\frac{\left.\lambda_{3}{ }^{4}-2 x \lambda^{3}{ }_{3}+3 x \lambda^{2}{ }_{3}+x^{2} \lambda^{2}{ }_{3}-3 x^{2} \lambda_{3}+2 x^{2}\right)\left(\lambda^{2}{ }_{3}-x \lambda_{3}+3 x\right)}{\left(\lambda_{3}+x\right)} \\
\equiv 0 \bmod \left(\lambda_{3}+2 x\right)
\end{gathered}
$$

Hence, equation (34) is a positive integer

$$
\frac{\left(\lambda_{3}{ }^{4}-2 x \lambda^{3}{ }_{3}+3 x \lambda^{2}{ }_{3}+x^{2} \lambda^{2}{ }_{3}-3 x^{2} \lambda_{3}+2 x^{2}\right)\left(y \lambda^{2}{ }_{3}-x \lambda_{3}+3 x\right)}{\left(\lambda_{3}+x\right)\left(\lambda_{3}+2 x\right)}
$$

Thus $\lambda_{3}$ takes any of the following values 2 and 4 . But $\lambda_{3}$ must be greater than 2 , hence 2 is not a possibility. We give corresponding values of $\lambda_{1}, \lambda_{2}, v, k$ and $b$ in the table below.

Table 25. Case 2; for $x=2$ the possible case of $4-(v, k, 1)$ design.

\begin{tabular}{lllllll}
\hline$\lambda_{3}$ & $\lambda_{2}$ & $\lambda_{1}$ & $k$ & $\boldsymbol{v}$ & $\boldsymbol{b}$ \\
\hline 4 & 10 & 20 & 4 & 7 & 35 \\
\hline
\end{tabular}

This $4-(7,4,1)$ design is trivial. Hence, for $x=2$ there is no nontrivial $4-(v, k, 1)$ design.

For $x=3, \lambda_{3}$ takes only 6 as its value. The corresponding values of $\lambda_{1}, \lambda_{2}, v, k$ and $b$ are given in the table 26 below.
Table 26. Case 2; for $x=3$ the possible case of $4-(v, k, 1)$ design.

\begin{tabular}{llllll}
\hline$\lambda_{3}$ & $\lambda_{2}$ & $\lambda_{1}$ & $\boldsymbol{k}$ & $\boldsymbol{v}$ & $\boldsymbol{b}$ \\
\hline 6 & 21 & 56 & 4 & 9 & 126 \\
\hline
\end{tabular}

This $4-(9,4,1)$ design is trivial. Hence, also for $x=3$ there is no nontrivial $4-(v, k, 1)$ design.

For $x=4, \lambda_{3}$ takes the values 8 and 28 . The corresponding values of $\lambda_{1}, \lambda_{2}, v, k$ and $b$ are given in the table below.

Table 27. Case 2; for $x=4$ the possible cases of $4-(v, k, 1)$ designs.

\begin{tabular}{llllll}
\hline$\lambda_{3}$ & $\lambda_{2}$ & $\lambda_{1}$ & $\boldsymbol{k}$ & $\boldsymbol{v}$ & $\boldsymbol{b}$ \\
\hline 8 & 36 & 120 & 4 & 11 & 330 \\
28 & 676 & 14365 & 9 & 171 & 272935 \\
\hline
\end{tabular}

We obtain the desired $4-(v, k, 1)$ designs from BIB designs below: $2-(11,4,36), 2-(171,9,676)$

For $x=5$ and using similar arguments as before, the possible values of $\lambda_{3}$ are as follows: 10,15 and 20 which gives the values of $\lambda_{1}, \lambda_{2}, v, k$ and $b$ as in the table below.

Table 28. Case 2; for $x=5$ the possible cases of $4-(v, k, 1)$ designs.

\begin{tabular}{llllll}
\hline $\boldsymbol{\lambda}_{\mathbf{3}}$ & $\boldsymbol{\lambda}_{\mathbf{2}}$ & $\boldsymbol{\lambda}_{\mathbf{1}}$ & $\boldsymbol{k}$ & $\boldsymbol{v}$ & $\boldsymbol{b}$ \\
\hline 10 & 55 & 220 & 4 & 13 & 715 \\
15 & 155 & 1240 & 5 & 33 & 8184 \\
20 & 305 & 3782 & 6 & 63 & 39711 \\
\hline
\end{tabular}

Case $3, x \neq y x>1, y>1$

In this case Equation (28) can be rewritten as

$$
\begin{gathered}
y \lambda_{2}=x(v-2), \Rightarrow v=\frac{y \lambda_{2}+2 x}{x} \text { and } y \lambda_{3}=x(k-2), \Longrightarrow \\
k=\frac{y \lambda_{3}+2 x}{x}
\end{gathered}
$$

Using Equation (28) we have

$\lambda_{1}=\frac{y^{2} \lambda_{3}{ }^{4}-2 x y \lambda^{3}{ }_{3}+3 x y \lambda^{2}{ }_{3}+x^{2} \lambda^{2}{ }_{3}-3 x^{2} \lambda_{3}+2 x^{2}}{y^{2} \lambda_{3}+x y}$

Which implies

$$
v=\frac{y \lambda_{3}{ }^{2}-x \lambda_{3}+3 x}{x} \text { and } k=\frac{y \lambda_{3}+2 x}{x}
$$

For this design to be $4-(v, k, 1)$ and from $b k=v \lambda_{1}$ it means

$\frac{\left.y^{2} \lambda_{3}{ }^{4}-2 x y \lambda^{3}{ }_{3}+3 x y \lambda^{2}{ }_{3}+x^{2} \lambda^{2}{ }_{3}-3 x^{2} \lambda_{3}+2 x^{2}\right)\left(y \lambda^{2}{ }_{3}-x \lambda_{3}+3 x\right)}{\left(y^{2} \lambda_{3}+x y\right)}$

That is Equation (38) is a positive integer

$$
\frac{\left(y^{2} \lambda_{3}{ }^{4}-2 x y \lambda^{3}{ }_{3}+3 x y \lambda^{2}{ }_{3}+x^{2} \lambda^{2}{ }_{3}-3 x^{2} \lambda_{3}+2 x^{2}\right)\left(y \lambda^{2}{ }_{3}-x \lambda_{3}+3 x\right)}{\left(y^{2} \lambda_{3}+x\right)\left(y \lambda_{3}+2 x\right)}
$$

Using similar argument as before Equation (38) will be integer if Equation (39) is an integer

$$
\frac{6 x^{3} y^{4}+216 x^{5} y^{2}+66 x^{4} y^{3}+216 x^{6} y}{y^{5}\left(y \lambda_{3}+x\right)\left(y \lambda_{3}+2 x\right)}
$$

The corresponding values of $\lambda_{1}, \lambda_{2}, v, k$ and $b$ is given in the table below. 
Table 29. Case 3; for $x=3$ and $y=2$ the possible case of $4-(v, k, 1)$ design.

\begin{tabular}{llllll}
\hline$\lambda_{3}$ & $\lambda_{2}$ & $\lambda_{1}$ & $\boldsymbol{k}$ & $\boldsymbol{v}$ & $\boldsymbol{b}$ \\
\hline 3 & 6 & 10 & 4 & 6 & 15 \\
\hline
\end{tabular}

This $4-(6,4,1)$ design is trivial. Hence, for $x=$ 3 andy $=2$ there is no nontrivial $4-(v, k, 1)$ design

Also for $x=5$ and $y=2$ there is no non-trivial $4-$ $(v, k, 1)$, the following table gives the corresponding values of $\lambda_{1}, \lambda_{2}, v, k$ and $b$.

Table 30. Case 3; for $x=5$ and $y=2$ the possible case of $4-(v, k, 1)$ design.

\begin{tabular}{llllll}
\hline$\lambda_{3}$ & $\lambda_{2}$ & $\lambda_{1}$ & $\boldsymbol{k}$ & $\boldsymbol{v}$ & $\boldsymbol{b}$ \\
\hline 5 & 6 & 35 & 4 & 8 & 70 \\
\hline
\end{tabular}

In this case there is only one non- trivial $4-(v, k, 1)$. The following table gives the corresponding values of $\lambda_{1}, \lambda_{2}, v, k$ and $b$.

Table 31. Case 3; for $x=4$ and $y=3$ the possible case of $4-(v, k, 1)$ design.

\begin{tabular}{llllll}
\hline$\lambda_{3}$ & $\lambda_{2}$ & $\lambda_{1}$ & $\boldsymbol{k}$ & $\boldsymbol{v}$ & $\boldsymbol{b}$ \\
\hline 4 & 12 & 30 & 5 & 11 & 66 \\
\hline
\end{tabular}

For $x=6$ and $y=3$ equation (28) becomes

$$
\frac{196560}{\left(3 \lambda_{3}+6\right)\left(3 \lambda_{3}+12\right)}
$$

And the corresponding values of $\lambda_{1}, \lambda_{2}, v, k a n d b$ are as shown below.

Table 32. Case 3; for $x=6$ and $y=3$ the possible cases of $4-(v, k, 1)$ designs.

\begin{tabular}{llllll}
\hline $\boldsymbol{\lambda}_{3}$ & $\boldsymbol{\lambda}_{2}$ & $\boldsymbol{\lambda}_{\mathbf{1}}$ & $\boldsymbol{k}$ & $\boldsymbol{v}$ & $\boldsymbol{b}$ \\
\hline 4 & 10 & 20 & 4 & 7 & 35 \\
6 & 26 & 91 & 5 & 15 & 273 \\
8 & 50 & 260 & 6 & 27 & 1170 \\
10 & 82 & 574 & 7 & 43 & 3526 \\
22 & 442 & 8177 & 13 & 223 & 140267 \\
\hline
\end{tabular}

For $x=7$ andy $=3$ equation (3.4.3.2) becomes

$$
\frac{466480}{\left(3 \lambda_{3}+7\right)\left(3 \lambda_{3}+14\right)}
$$

In this case there is only one non- trivial $4-(v, k, 1)$. The following table gives the corresponding values of $\lambda_{1}, \lambda_{2}, v, k$ and $b$.

Table 33. Case 3; for $x=7$ and $y=3$ the possible case of $4-(v, k, 1)$ design.

\begin{tabular}{llllll}
\hline$\lambda_{3}$ & $\lambda_{2}$ & $\lambda_{1}$ & $\boldsymbol{k}$ & $\boldsymbol{v}$ & $\boldsymbol{b}$ \\
\hline 7 & 35 & 140 & 5 & 17 & 476 \\
\hline
\end{tabular}

For $x=8$ and $y=3$ equation (3.4.3.2) becomes

$$
\frac{992256}{\left(3 \lambda_{3}+8\right)\left(3 \lambda_{3}+16\right)}
$$

Also in this case, there is only one non- trivial $4-$ $(v, k, 1)$. The following table gives the corresponding values of $\lambda_{1}, \lambda_{2}, v, k$ and $b$.

Table 34. Case 3; for $x=8$ and $y=3$ the possible case of $4-(v, k, 1)$ design.

\begin{tabular}{llllll}
\hline$\lambda_{3}$ & $\lambda_{2}$ & $\lambda_{1}$ & $\boldsymbol{k}$ & $\boldsymbol{v}$ & $\boldsymbol{b}$ \\
\hline 40 & 1496 & 52547 & 17 & 563 & 1740233 \\
\hline
\end{tabular}

For $x=9$ and $y=3$ equation (3.4.3.2) becomes

$$
\frac{1939140}{\left(3 \lambda_{3}+9\right)\left(3 \lambda_{3}+18\right)}
$$

And the corresponding values of $\lambda_{1}, \lambda_{2}, v, k$ and $b$ are as shown below.

Table 35. Case 3; for $x=9$ and $y=3$ the possible cases of $4-(v, k, 1)$ designs.

\begin{tabular}{llllll}
\hline$\lambda_{\mathbf{3}}$ & $\boldsymbol{\lambda}_{\mathbf{2}}$ & $\boldsymbol{\lambda}_{\mathbf{1}}$ & $\boldsymbol{k}$ & $\boldsymbol{v}$ & $\boldsymbol{b}$ \\
\hline 6 & 21 & 56 & 4 & 9 & 126 \\
9 & 57 & 285 & 5 & 21 & 1197 \\
15 & 183 & 1891 & 7 & 63 & 17019 \\
\hline
\end{tabular}

\section{Conclusion}

In this study a new recursive technique has been developed for the construction of $t-\left(v, k, \lambda_{t}\right)$ designs. Thus, the study has presented an alternative method that is simpler and unified for the construction of BIBDs that are very important in the experimental designs. As it provides designs for different values of $k$, unlike many methods that provide designs for a single value of $k$. More so, it provides both Steiner and non-Steiner designs.

\section{Recommendations}

Although this study has provided a technique for the construction of $t$ - designs, it is still clear that construction method of $t-$ designs is not known in general. In fact, it is not clear how one might construct $t$-designs with arbitrary block size. We therefore invite researchers to come up with "additive theorems "for this construction to make it general for any value of $t$ as this may bring in new techniques and ideas. There is also need for obtaining a theorem which would give all values of $x$ and $y$ for the case three in this construction in order to see new Steiner $t$-designs. Lastly, if there is a computer package that could be incorporated in the method to aid in calculations.

\section{References}

[1] Blanchard, J. L (1995a). A construction for Steiner 3-designs, Journal of combinatorial Theory A, 71, 60-67.

[2] Blanchard, J. L. (1995b). An extension theorem for Steiner systems, Discrete Mathematics 141, no. 1-3, 23-35.

[3] Blanchard, J. L (1995c). A construction for orthogonal arrays with strength $\mathrm{t} \geq 3$, Discrete math 137 , no. 1-3, 35-44.

[4] Bogart, K. P. (1990). Introductory Combinatorics second edition, Harcourt Brace Bose, Jovanovich, Inc. Orlando, Florida. 
[5] Cameron, P. J., Maimani, H. R., Omidi, G. R., and TayfehRezaie, B. (2006). 3-designs PGL (2, q), Discrete Mathematics, 306, vol.23, 3063-3073.

[6] Colbourn, C. J. et al. (2002). Orthogonal arrays of strength three from regular 3-wise balanced designs.

[7] Dinitz, J. H., \& Stinson, D. R. (1992). Contemporary Design Theory: A collection ofsurveys, Wiley-Interscience.

[8] Hartman, A. (1994). The fundamental construction for 3designs. Discrete math 124, no.1-3, 107-131.

[9] Magliveras, S. S., Kramer, E. S., \& Stinson, D. R. (1993). Some new Large sets of t-designs. Australasian journal of Combinatorics 7, pp 189-193.

[10] Mohácsy, H., and Ray-Chaudhuri, D. K. (2001). A construction for infinite families of Steiner 3-designs, Journal of Combinatorial Theory A, 94, 127-141.
[11] Mohácsy, H., and Ray-Chaudhuri, D. K. (2002). Candelabra Systems and designs, Journalof Statistical planning and Inference, 106, 419-448.

[12] Mohácsy, H., and Ray-Chaudhuri, D. K. (2003). A construction for group divisible $t$-designs with strength $t \geq 2$ and index unity, Journal of Statistical planning and Inference, 109, 167-177.

[13] Onyango, O. F. (2010). Construction of $\mathrm{t}-\left(\mathrm{v}, \mathrm{k}, \lambda_{\mathrm{t}}\right)$ designs, Journal of mathematical science, vol. 21 no. 4 pp 521-526.

[14] Qiu-rong Wu. (1991). A note on extending t-designs, Australasia. Journal of Combinatorics, 4 pp 229-235.

[15] Stinson, D. R. (2004). Combinatorial Designs: Construction and Analysis, Springer_Verlag, New York, Inc., New York. 